\title{
Efficient Global Threshold Vector Outlyingness Ratio Filter for the Removal of Random Valued Impulse Noise
}

\author{
J. Amudha1*, R. Sudhakar ${ }^{2}$ \\ ${ }^{1}$ Department of Electrical and Electronics Engineering, Dr. Mahalingam College of Engineering and Technology, \\ Pollachi, India \\ ${ }^{2}$ Department of Electronics and Communication Engineering, Dr. Mahalingam College of Engineering and \\ Technology, Pollachi, India

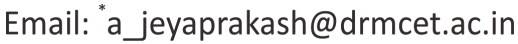

Received 9 March 2016; accepted 7 May 2016; published 11 May 2016

Copyright @ 2016 by authors and Scientific Research Publishing Inc. This work is licensed under the Creative Commons Attribution International License (CC BY). http://creativecommons.org/licenses/by/4.0/

(c) (i) Open Access

\section{Abstract}

This research paper proposes a filter to remove Random Valued Impulse Noise (RVIN) based on Global Threshold Vector Outlyingness Ratio (GTVOR) that is applicable for real time image processing. This filter works with the algorithm that breaks the images into various decomposition levels using Discrete Wavelet Transform (DWT) and searches for the noisy pixels using the outlyingness of the pixel. This algorithm has the capability of differentiating high frequency pixels and the "noisy pixel" using the threshold as well as window adjustments. The damage and the loss of information are prevented by means of interior mining. This global threshold based algorithm uses different thresholds for different quadrants of DWT and thus helps in recovery of noisy image even if it is $\mathbf{9 0 \%}$ affected. Experimental results exhibit that this method outperforms other existing methods for accurate noise detection and removal, at the same time chain of connectivity is not lost.

\section{Keywords}

Image Restoration, Noise Detection, Noise Removal, Random Valued Impulse Noise, Global Threshold Vector Outlyingness Ratio

\section{Introduction}

Images are often corrupted by impulse noise because of sensors or channel transmission [1]. Impulse noise is ${ }^{*}$ Corresponding author. 
classified as fixed value impulse noise and random valued impulse noise. Generally the impulsive noise such as salt and pepper noise has the nature of producing the highest pixel value (255) and the lowest pixel value (zero) in an eight bit image. This nature provides the filtering algorithm to point out possibilities of the noise and has to spend only limited computation power to identify exact "noise free" pixels. The problem in real time images is that the salt and pepper noise may not occur in the form as mentioned above. Instead of pixel value 255 there may be the value of 250 , which is still a salt noise. Instead of pixel value 0 there may be value 10 , which is still a pepper noise. The existing algorithms will find difficulties in processing and removing this noise when the above mentioned change occurred in an image.

In Gaussian filter [2], the Euclidean distance between the current pixel and its neighborhood is calculated. In median filter, each pixel is replaced by the median value in its neighborhood, and thus destroys the significant information in the image. To overcome this, weighted-based median filters [3] are proposed. However, like median filter, the drawback of weighted-based median filters [4] is that they replace each pixel with weighted median value in its neighborhood regardless of "noisy" or "noise free" pixel. The switching-based median filters have been proposed to detect "noisy" pixels replaced by median value, whereas the "noise free" pixels are left unchanged, for example, Adaptive Switching Median Filter (ASMF) [5]. However, these filters use median values or their variations to recover the "noisy" pixels, but they introduce blur in the image details [6].

To overcome this problem, edge-preserving regularization filters are introduced by employing two-stages. In the first stage, the "noisy" pixels are identified by a noise detector. In the second stage, the "noisy" pixels are recovered by an edge preserving regularization term without affecting the edges and "noise free" pixels which have to be preserved. Papers [7]-[11] show that the noise removing capability depends on the accuracy of the noise detector in these two stage methods. To this end, this paper will employ a better noise detector for twostage method. Local outlier-based impulse noise detectors are proposed in papers [12] recently. Local Outlier Factor (LOF) and Robust Outlyingness Ratio (ROR) are employed to identify the fixed-valued impulse noise and to measure the outlyingness of each and every pixel in the image respectively. Normally ROR has the capability of giving the outlyingness, but it always finds the outlyingness with fixed threshold [13] [14]. A global threshold is used to find the outlyingness based on selecting better threshold for the data given. In this paper a single algorithm is proposed using Global Threshold Vector Outlyingness Ratio (GTVOR) to detect the noise as well as to remove the noise. This technique uses the impulse-free information to recover the image.

\section{Noise Detection and Removal}

\subsection{Noise Model}

In fixed value impulse noise, "noisy" pixels take either minimum or maximum values i.e., $\eta F(i, j) \in\left\{N_{\min }\right.$, $\left.N_{\max }\right\}$ where $N_{\min }=0$ and $N_{\max }=255$, whereas Random Variable impulse noise,(RVIN) "noisy" pixels take any value within the minimum range to maximum range i.e., $\eta V(i, j) \in\left[N_{\min }, N_{\max }\right]$, where $N_{\min }=[0, l]$, and $N_{\max }=$ [255 - l, 255] denote the minimum range and maximum range. Consequently, removal of variable type impulse noise is not easy compared to the removal of fixed value impulse noise. Let us consider the random valued impulse noise model,

$$
u_{x, y}=\left\{\begin{array}{l}
{\left[N_{\min }\right], \text { with probability } p} \\
{\left[N_{\max }\right], \text { with probability } q} \\
o_{x, y}, \text { with probabiliy }(1-(p+q))
\end{array}\right.
$$

where,

$p$ is the probability of noise whose values fall in the range of $[0, l]$,

$q$ is the probability of noise whose values fall in the range of [(255 - l), 255],

noise probability $r=p+q$ and $p=q$,

$o_{x, y}$ and $u_{x, y}$ is the current pixel value of original and noisy image at coordinate $(x, y)$.

\subsection{Proposed Method}

Let $o=\{o(x, y), x, y=1,2, \cdots, M\}$ denote $M \times M$ original image to be recovered, $M$ is integer power of 2 . 
RVIN is introduced during the signal acquisition stage the original image I gets corrupted. The noisy observation $u(x, y)=o(x, y)+\sigma n(x, y)$ is obtained. To recover $o(x, y)$ from $u(x, y)$ is our aim, such that the Mean square Error (MSE) is less. DWT of $u$ is matrix of wavelet coefficients with 4 sub bands (LL, LH, HL, HH).The sub bands LH, HL and HH are detailed coefficients and LL represents approximation coefficients. We can perform DWT of approximation sub band multiple times until the final approximation band contains only single value. Denote the maximum number of decompositions by J. The size of the sub band at scale $k$ is $M / 2^{k} \times M / 2^{k}$. Figure 1 represents two level decomposition of an image.

Figure 2 depicts the process carried out in removing the noise and recovering the de-noised image from the noisy image.

Discrete Wavelet Transform is applied for the noisy image under consideration. The decomposition level can be increased depending upon the noise level. DWT can slice the frequencies available in the image and able to give them as spatial co-ordinates for processing. The coefficient of LL, LH, HL, HH are let in to further processing. Separate the wavelet coefficients into Small Blocks with $(2 \mathrm{~N}+1) \times(2 \mathrm{~N}+1)$ window (for $\mathrm{N}=1,3 \times$ 3 ) for the all four Coefficients i.e. LL, LH, HL, HH. Let the $3 \times 3$ window of wavelet coefficient be $\mathrm{X}=\left[\mathrm{X}_{1}, \mathrm{X}_{2}, \mathrm{X}_{3}, \mathrm{X}_{4}, \mathrm{X}_{5}, \mathrm{X}_{6}, \mathrm{X}_{7}, \mathrm{X}_{8}, \mathrm{X}_{9}\right]$, For this data $\mathrm{X}$ compute GTVOR by computing the Median(MED), Median Absolute Difference(MAD), and Global Threshold Interior Mining (GTIM) factor using the Equations (2)-(6). Global Threshold (GT) is computed using the algorithm.

$$
\begin{gathered}
\operatorname{MED}=\operatorname{Median}(\mathrm{X}) \\
\operatorname{MAD}=\operatorname{Median}(\operatorname{Absolute}(\mathrm{X}-\mathrm{MED})) \\
\text { GTIM }=\frac{\mathrm{GT}}{10^{3} / 10^{\text {pass- }}}
\end{gathered}
$$

\begin{tabular}{|c|c|c|}
\hline $\mathrm{LL}_{2}$ & $\mathrm{HL}_{2}$ & \multirow{2}{*}{$\mathrm{HL}_{1}$} \\
\cline { 1 - 2 } $\mathrm{LH}_{2}$ & $\mathrm{HH}_{2}$ & \\
\hline & & \\
\hline \multicolumn{2}{|c|}{$\mathrm{LH}_{1}$} & $\mathrm{HH}_{1}$ \\
& & \\
\hline
\end{tabular}

Figure 1. Two-dimensional DWT with level-2 decomposition of an image.
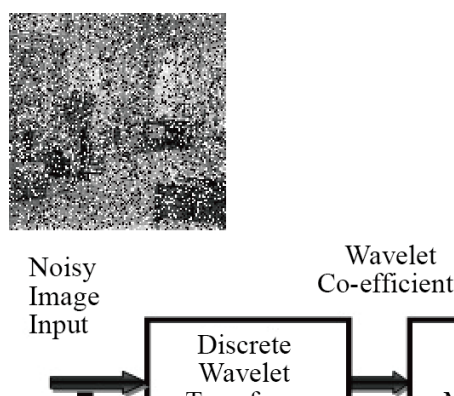
Co-efficients

Transform o-efficients

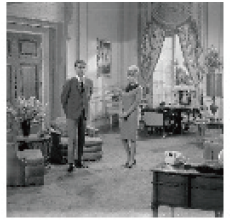

De-noised

Image 


$$
\begin{gathered}
\text { MADN }=\frac{\text { MAD }}{\text { GTIM }} \\
\text { GTVOR }=\text { Absolute }\left[\frac{X-\text { MED }}{\text { MADN }}\right]
\end{gathered}
$$

Let GTVOR $=\left[\mathrm{GX}_{1}, \mathrm{GX}_{2}, \mathrm{GX}_{3}, \mathrm{GX}_{4}, \mathrm{GX}_{5}, \mathrm{GX}_{6}, \mathrm{GX}_{7}, \mathrm{GX}_{8}, \mathrm{GX}_{9}\right]$. The sign of each and every ratio should be noted and floored. For example if $\mathrm{GX}_{3}$ is greater than Th (Threshold), then the corresponding coefficient $\mathrm{X}_{3}$ will be suspected as noisy. The corresponding noisy pixel is replaced by the average of the noise free neighbors. This is done in spatial image. Hence we need not to use inverse transform. If the noisy pixel is not detected in this pass, the pass is incremented for further noise mining process. The iterative process of this filtering is done to remove the noise until the GTVOR detects no outlyingness. Thus the noise free image is recovered. Figure 3 will provide the clarity in recovering the de-noised image in steps.

\section{Algorithm to compute GT}

a. Compute histogram and probabilities of each intensity level

b. Initialize $\omega_{i}(0)$ and $\mu_{i}(0)$

c. For all possible thresholds $\mathrm{T}=1 \ldots$ maximum intensity

i. Update $\omega_{\mathrm{i}}$ and $\mu_{\mathrm{i}}$

ii. Compute $\sigma_{b 2}(\mathrm{~T})$

d. Desired threshold corresponds to the maximum $\sigma_{\mathrm{b} 2}(\mathrm{~T})$

e. Compute two thresholds $\sigma_{\mathrm{b} 12}(\mathrm{~T})$ is the threshold $($ greater max $)$ and $\sigma_{\mathrm{b} 22}(\mathrm{~T})$ is the threshold 2 (greater or equal maximum)

f. $\quad$ Desired Threshold $=\frac{\text { threshold }_{1}+\text { threshold }_{2}}{2}$

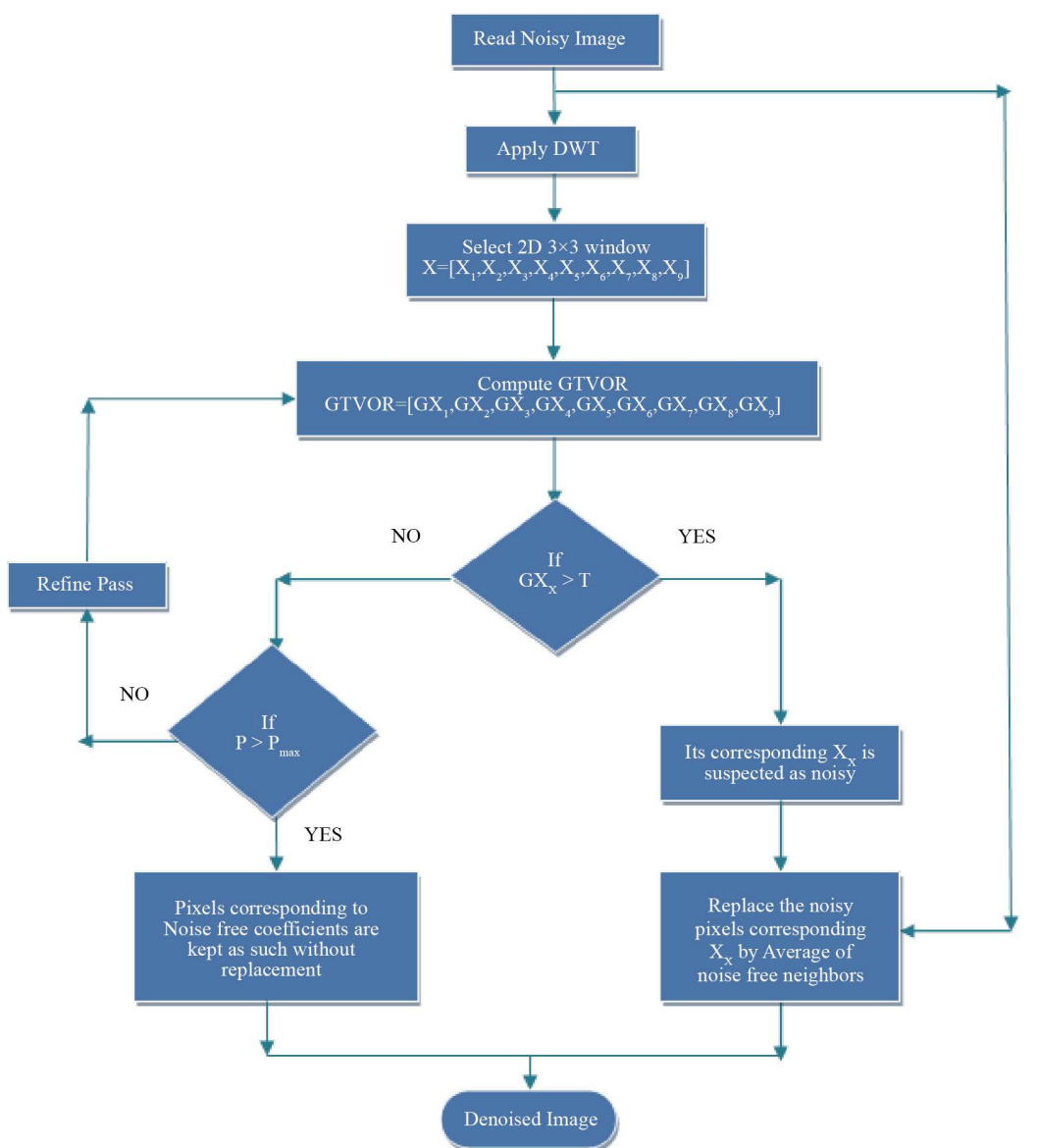

Figure 3. Flowchart. 


\section{Simulation Results}

Standard test images of size $512 \times 512$ such as Living Room Image, Boat image, Lena Image and Gorilla image are taken from data set USC-SIPI Image Database. GTVOR algorithm is evaluated and compared with many other existing filters. For performance comparison, the Wiener filter, Median filter, Noise Adaptive Switched Median filter and Proposed GTVOR filter have been simulated by programming models and the results were tabulated in Table 1 for $80 \%$ of random valued impulse noise. The image outputs produced by various filters were compared with proposed GTVOR filter with 70\% noise was presented in Figure 4.
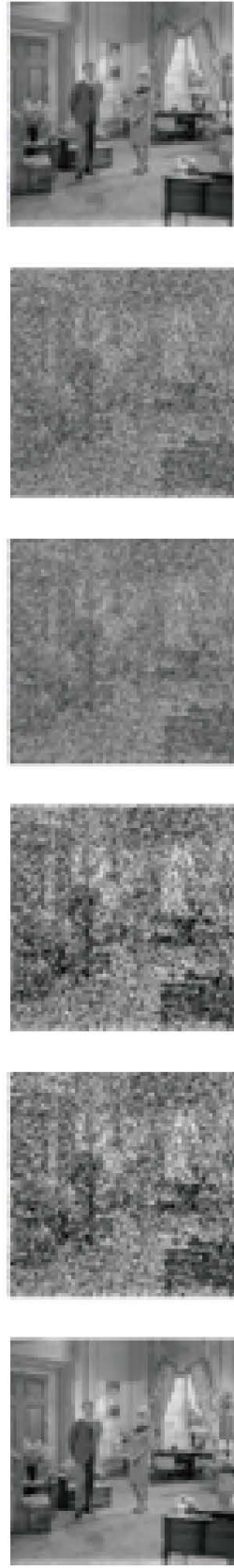
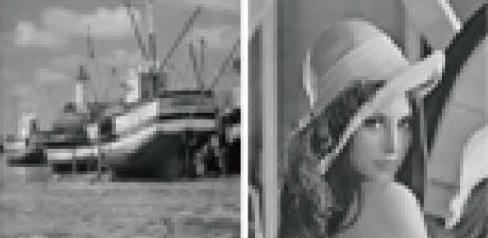

(a)

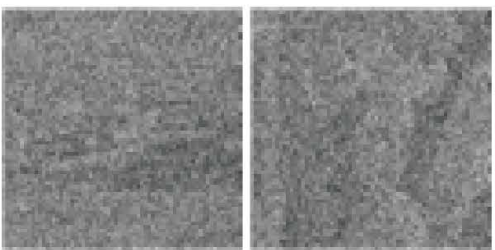

(b)

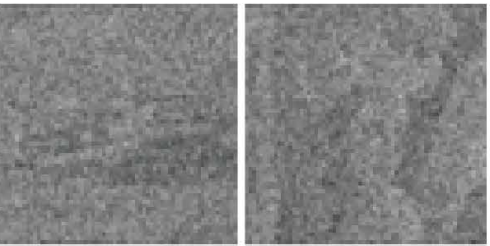

(c)

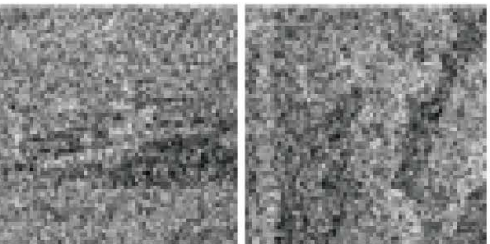

(d)
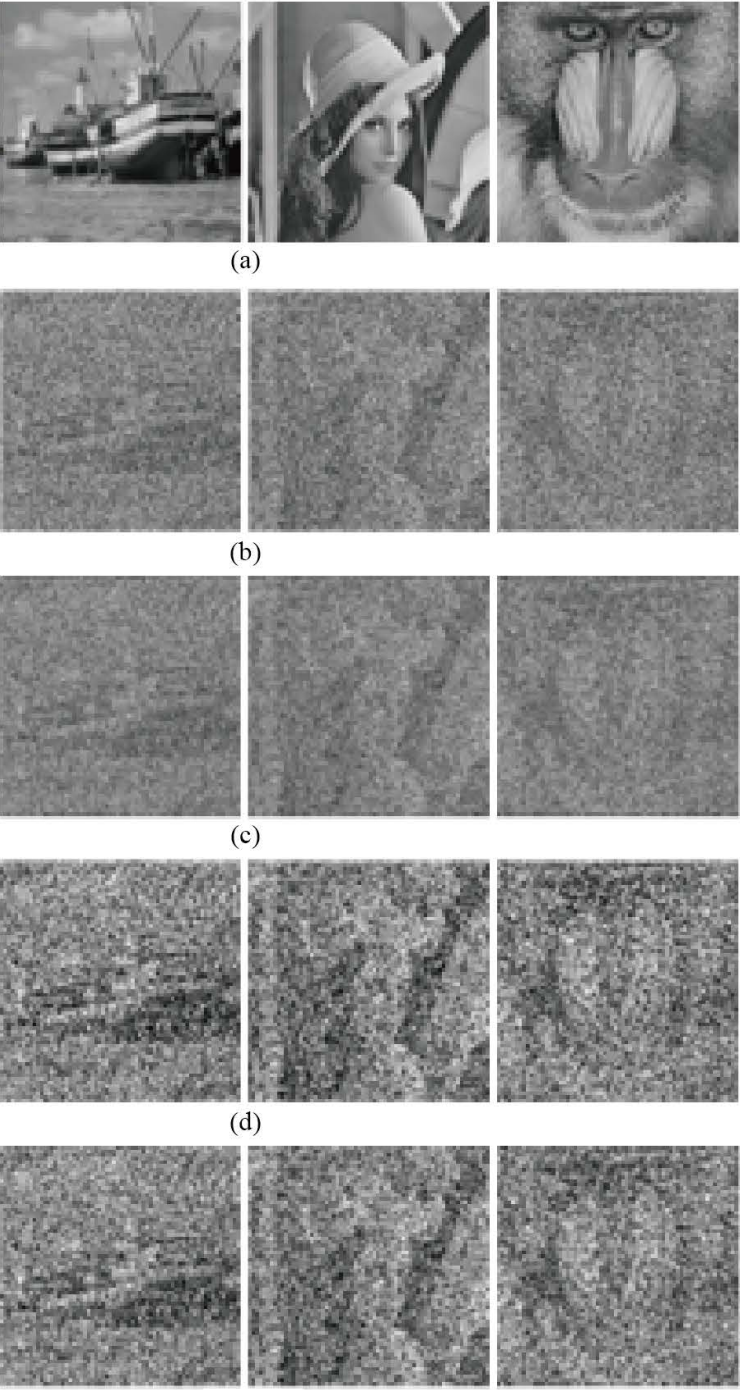

(e)

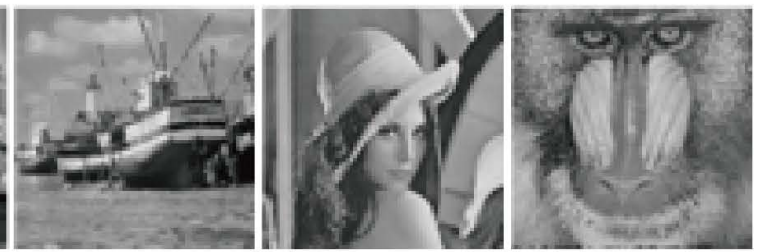

(f)

Figure 4. (a) Noise-free test images of size $512 \times 512$; (b) $70 \%$ noisy images; (c) Restored images by Wiener Filter; (d) Restored images by Median Filter; (e) Restored images by Adaptive Switched Median Filter; (f) Restored images by proposed GTVOR Filter. 
Table 1. Results in MSE, PSNR and SNR after filtering images corrupted by 80\% Random valued impulse noise.

\begin{tabular}{ccccccc}
\hline $\begin{array}{c}\text { Name } \\
\text { of Image }\end{array}$ & $\begin{array}{c}\text { Image Quality } \\
\text { Parameters }\end{array}$ & $\begin{array}{c}80 \% \\
\text { Noisy image }\end{array}$ & Wiener Filter & Median Filter & ASMF & $\begin{array}{c}\text { Proposed } \\
\text { GTVOR Filter }\end{array}$ \\
\hline $\begin{array}{c}\text { Living Room } \\
\text { Image }\end{array}$ & MSE & $14,616.90$ & 1997.87 & 9862.97 & 9898.38 & 256.58 \\
& PSNR & 6.48 & 15.13 & 8.19 & 8.18 & 24.04 \\
Boat Image & SNR & 0.64 & 2.30 & 2.26 & 2.22 & 6.78 \\
& MSE & $14,746.87$ & 2082.65 & 9937.45 & 9976.38 & 238.32 \\
& PSNR & 6.44 & 14.94 & 8.16 & 8.14 & 24.36 \\
Lena Image & SNR & 1.42 & 3.69 & 3.02 & 2.99 & 8.04 \\
& MSE & $14,860.85$ & 2138.40 & 9995.45 & $10,077.53$ & 124.69 \\
& PSNR & 6.41 & 14.83 & 8.13 & 8.10 & 27.17 \\
Gorilla Image & SNR & 0.93 & 2.13 & 2.45 & 2.33 & 8.98 \\
& MSE & $14,423.72$ & 1906.30 & 9901.85 & 9943.86 & 589.32 \\
\hline
\end{tabular}

Through this comparison it is clear that the proposed GTVOR filter is the best among these. The reason behind is the existing filters has no capability of differentiating "noisy" pixel among the group, and blindly process all the pixels. Existing filters were explored fully on the spatial domain of the image and implemented identically across the image, they tend to modify irrespective of noisy and noise free pixels and removes desirable details, too.

Our proposed algorithm break the images into various decomposition level and searches for the anomaly/odd pixels by which the capability of noise detector is accurate than existing algorithms. Certain algorithms such as median filter has the capability of selecting the window to process, but the capability of adjusting the high frequency noise by using its own threshold value is not handled in the switching sequence. These algorithms were flexible only up to certain limit to use mean, median and mode operations. The ability to differentiate high frequency pixels and the "noisy pixels" using the thresholds is the best highlight of the proposed technique. The damage and the loss of information is prevented by means of interior mining capability of this algorithm. The inner mining will be more effective with bonded type noise since the proposed algorithm uses global threshold for each window in process, which makes the algorithm adaptive to any image irrespective of nature of input.

The proposed GTVOR filter is tested for $512 \times 512$ Living room image. The performance of the proposed filter is tested for all levels of noise densities. Each time the test image is corrupted by random valued impulse noise of different density ranging from $10 \%$ to $90 \%$ with an increment of $10 \%$ and tested for removal of noise capability. The performance at various noise densities for living room image is shown in Table 2 and plotted in Figure 5 and Figure 6.

From Figure 5 and Figure 6, it is clear that Median filter and Adaptive Median filters perform better than Wiener filter when the noise level is less than $40 \%$ and $50 \%$ respectively. But at high noise densities the median of window itself have the effect of noise resulting in poor quality in restoring images. As Wiener filter have the behavior of Linear time invariant that estimates the spatial intensity values of the 2D signal through which the filtering or smoothening was done. At high noise levels the estimation values will also have the effect of noise and hence restoration is poor. As the proposed filter has the capability of computing various thresholds in different quadrants, noise detection is more accurate than all other existing filters. Comparison of histograms for $70 \%$ noisy "Living Room Image" restored by different filters is shown in Figure 7. The results are prominent and the reconstruction quality is better even at higher level of noise densities.

\section{Conclusions}

This paper proposes a new filter, named Global Threshold Vector Outlyingness Ratio (GTVOR) Filter that 

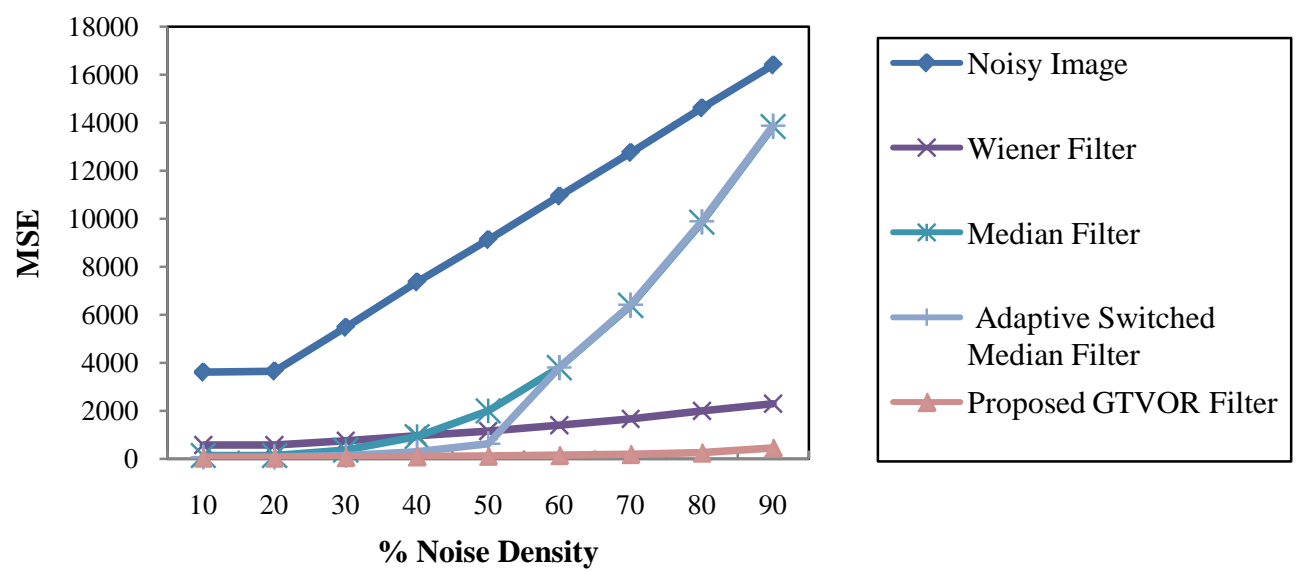

Figure 5. MSE values for different filters operating on the image "Living Room” at various noise densities.
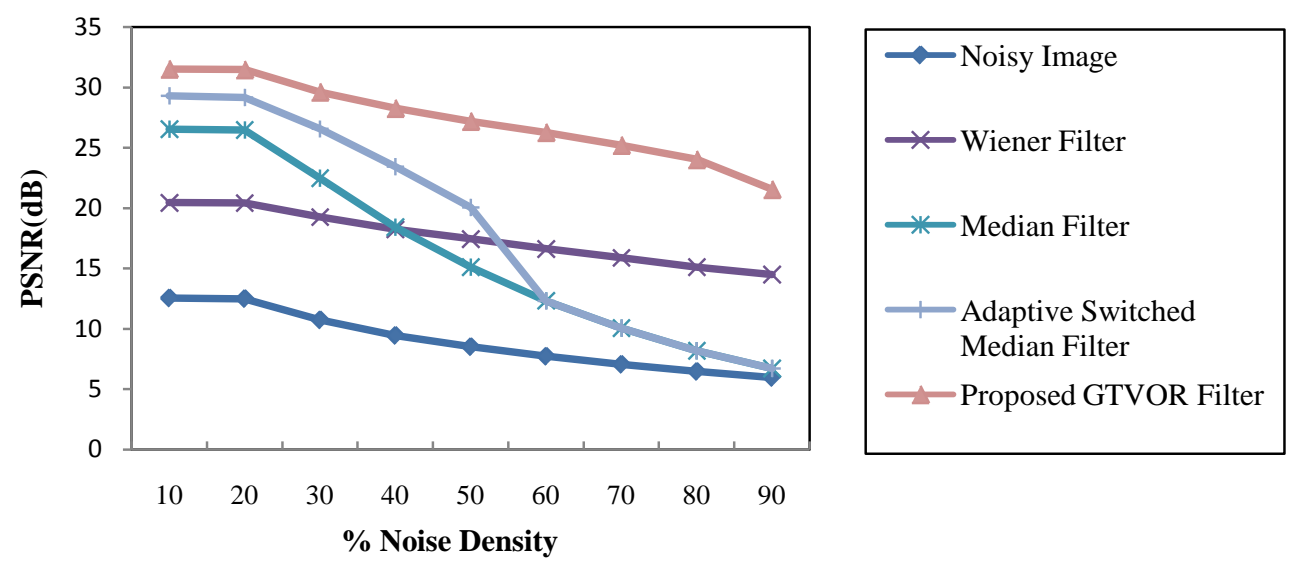

Figure 6. PSNR values for different filters operating on the image "Living Room” at various noise densities.

Table 2. PSNR in dB for different filters operating on the image "Living Room" at various noise densities.

\begin{tabular}{cccccc}
\hline Noise Density \% & Noisy Image & Weiner Filter & Median Filter & ASMF & Proposed GTVOR Filter \\
\hline 10 & 12.54696 & 20.45823 & 26.54639 & 29.30889 & 31.52968 \\
20 & 12.49474 & 20.43171 & 26.47796 & 29.15956 & 31.47864 \\
30 & 10.74031 & 19.27955 & 22.48193 & 26.56197 & 29.6299 \\
40 & 9.455607 & 18.25742 & 18.4182 & 23.42616 & 28.2796 \\
50 & 8.524303 & 17.45805 & 15.10965 & 20.04897 & 27.20809 \\
60 & 7.737345 & 16.63694 & 12.32869 & 12.318 & 26.28682 \\
70 & 7.071953 & 15.9051 & 10.06975 & 10.05166 & 25.22392 \\
80 & 6.482251 & 15.12513 & 8.190725 & 8.175163 & 24.03864 \\
90 & 5.980825 & 14.51399 & 6.715813 & 6.70808 & 21.56399 \\
\hline
\end{tabular}

works in two stages namely detecting stage and filtering stage to recover highly-corrupted images effectively, which can be used for many real-time image processing applications. The proposed GTVOR filter is capable of suppressing impulse noise even at high level of noise density (90\%), especially, without affecting the edges and textures. Extensive experimental results depict that the proposed GTVOR filter outperforms consistently compared with other filters. Comparing with the others existing filters, our filter has better image restoring capability with precise noise detection. 


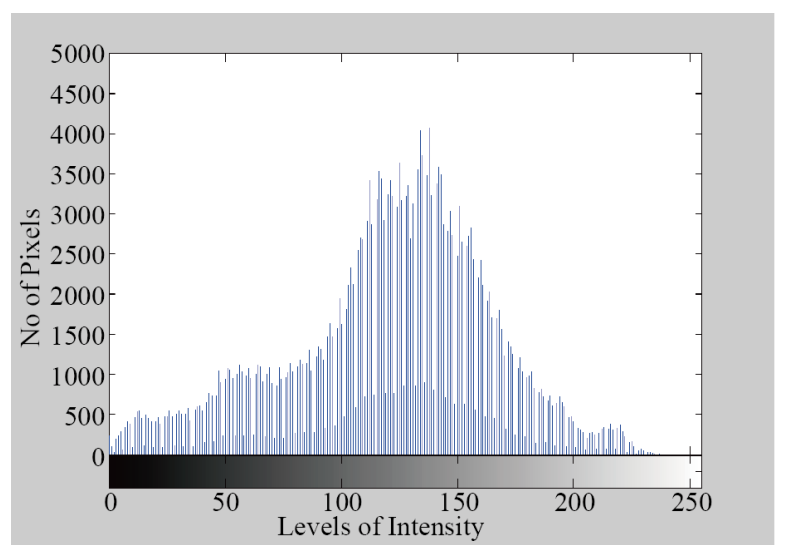

(a)

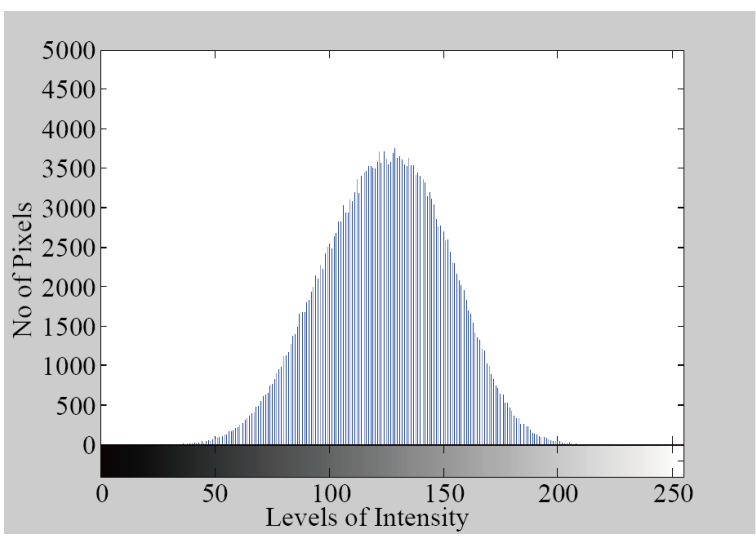

(c)

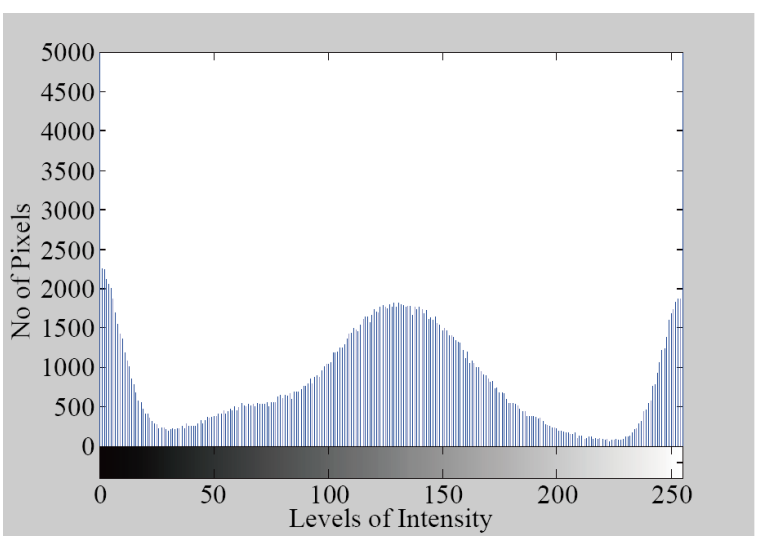

(e)

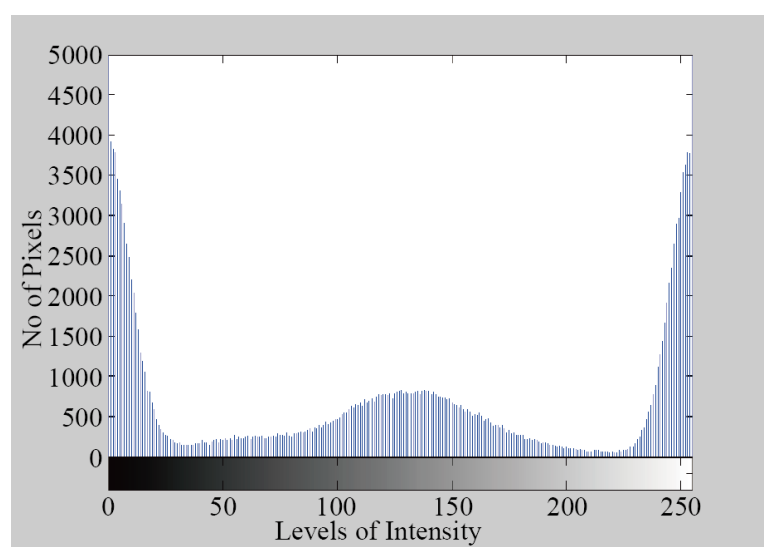

(b)

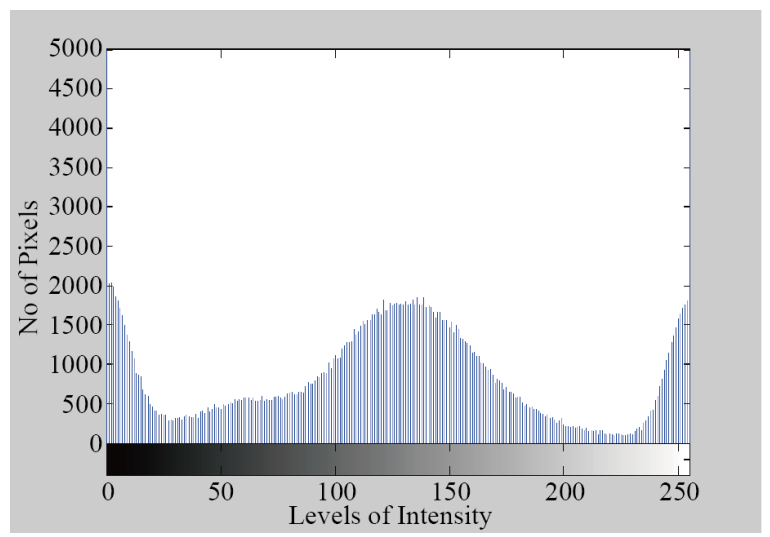

(d)

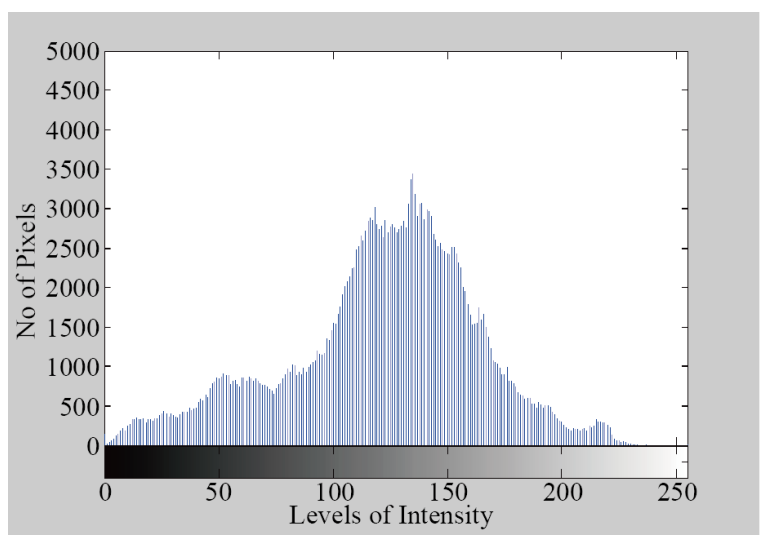

(f)

Figure 7. Comparison of histograms for $70 \%$ noisy "living room image” restored by different filters. (a) Noise free living room image; (b) 70\% noisy image; (c) Wiener filter output; (d) Median filter output; (e) Adaptive switching median filter output; (f) Proposed GTVOR filter output.

According to the current research the GTVOR filter perfectly works with 8 bit images, which is used for general purpose applications. This filter has some limitations while working with medical images. The medical field has the intensive requirement for image restoration and denoising, since the medical images such as DICOM, multispectral images may involve such noise. But these images are 16 bit and the combination of pixels varies from 0 to 65535 (65536 pixel values). Therefore it is a real challenge at window decomposition of the image and in computing the outlyingness. Also the information constrained with the global threshold value varies with large interval which should be addressed in the future. 


\section{References}

[1] Gonzalez, R.C. and Woods, R.E. (2002) Digital Image Processing. Prentice-Hall, Englewood Cliffs.

[2] Wang, Z. and Zhang, D. (1999) Progressive Switching Median Filter for the Removal of Impulse Noise from Highly Corrupted Images. IEEE Transactions on Circuits and Systems_II: Analog and Digital Signal Processing, 46, 78-80. http://dx.doi.org/10.1109/82.749102

[3] Brownrigg, D.R.K. (1984) The Weighted Median Filter. Communications of the ACM, 27, 807-818. http://dx.doi.org/10.1145/358198.358222

[4] Ko, S.J. and Lee, S.J. (1991) Center weighted Median Filters and Their Applications to Image Enhancement. IEEE Transactions on Circuits and Systems, 38, 984-993. http://dx.doi.org/10.1109/31.83870

[5] Akkoul.S, Ledee, R., Leconge, R. and Harba, R. (2010) A New Adaptive Switching Median Filter. IEEE Signal Processing Letters, 17,587-590. http://dx.doi.org/10.1109/LSP.2010.2048646

[6] Nikolova, M. (2004) Avariational Approach to Remove Outliers and Impulse Noise. Journal of Mathematical Imaging and Vision, 20, 99-120. http://dx.doi.org/10.1023/B:JMIV.0000011920.58935.9c

[7] Chan, R.H., Hu, C. and Nikolova, M. (2004) An Iterative Procedure for Removing Random-Valued Impulse Noise. IEEE Signal Processing Letters, 11, 921-924. http://dx.doi.org/10.1109/LSP.2004.838190

[8] Dong, Y., Chan, R.H. and Xu, S. (2007) A Detection Statistic for Random Valued Impulse Noise. IEEE Transactions on Image Processing, 16, 1112-1120. http://dx.doi.org/10.1109/TIP.2006.891348

[9] Chan, R.H., Ho, C.W. and Nikolova, M. (2005) Salt-and-Pepper Noise Removal by Median-Type Noise Detectors and Detail Preserving Regularization. IEEE Transactions on Image Processing, 14, 1479-1485.

[10] Huang, Y., Ng, M.K. and Wen, Y. (2009) Fast Image Restoration Methods for Impulse and Gaussian Noise Removal. IEEE Signal Processing Letters, 16, 457-460. http://dx.doi.org/10.1109/LSP.2009.2016835

[11] Allard, W.K. (2008) Total Variation Regularization for Image Denoising. SIAM Journal on Imaging Sciences, 1, 400-417. http://dx.doi.org/10.1137/070698749

[12] Wang, W. and Lu, P. (2011) An Efficient Switching Median Filter Based on Local Outlier Factor. IEEE Signal Processing Letters, 18, 551-554. http://dx.doi.org/10.1109/LSP.2011.2162583

[13] Xiong, B. and Yin, Z. (2012) A Universal Denoising Framework with A New Impulse Detector and Nonlocal Means. IEEE Transactions on Image Processing, 21, 1663-1675. http://dx.doi.org/10.1109/TIP.2011.2172804

[14] Breuig, M.M. (2000) LOF: Identifying Density-Based Local Outliers. Proceedings of the ACM SIGMOD Conference on Management of Data, Dallas, 15-18 May 2000, 93-104. http://dx.doi.org/10.1145/335191.335388 